\title{
Dynamics of Numerics of Nonautonomous Equations with Periodic Solutions: Introducing the Numerical Floquet Theory
}

\author{
Melusi Khumalo \\ Department of Mathematics, University of Johannesburg, P.O. Box 17011, Doornfontein 2028, South Africa \\ Correspondence should be addressed to Melusi Khumalo; mkhumalo@hotmail.com
}

Received 22 September 2012; Revised 2 March 2013; Accepted 26 March 2013

Academic Editor: Alberto Cabada

Copyright ( 2013 Melusi Khumalo. This is an open access article distributed under the Creative Commons Attribution License, which permits unrestricted use, distribution, and reproduction in any medium, provided the original work is properly cited.

\begin{abstract}
Nonautonomous systems with periodic solutions are encountered frequently in applications. In this paper, we will consider simple systems whose solutions are periodic with a known period. Their transformation under linearized collocation methods is investigated, using a technique called stroboscopic sampling, a discrete version of the well-known Poincaré map. It is shown that there is an inextricable relationship between AN stability (or BN stability) of the numerical methods and the correct qualitative behaviour of solutions.
\end{abstract}

\section{Introduction}

Let

$$
x^{\prime}=f(x, t), \quad x(0)=x_{0},
$$

where $f: I \subset \mathbb{R} \times \mathbb{R} \rightarrow \mathbb{R}$, be a scalar ordinary differential equation in which $f(x, t)$ is a periodic function of $t$ with prime period $T$.

The detailed dynamics of numerics for nonautonomous ODEs has notably been lacking. Although any nonautonomous ODE can be transformed to an autonomous one, thereby increasing the dimension by one, the familiar dynamics of autonomous equations which is centered around the notion of equilibrium points [1] is lost. In certain special cases, this notion is replaced by that of periodicity. It is on these special cases that we will focus our attention. Stuart [2] proved using the bifurcation theory that for reactiondiffusion-convection equations, linearized instability implies the existence of spurious periodic solutions. Our approach differs from that of Stuart, who considered partial differential equations. Nonautonomous ODEs where $f$ is periodic in $t$ are very common in applications such as population dynamics with seasonal parameters or periodically forced systems.

Under certain conditions on $f$, (1) has a unique $T$ periodic solution [3]. We will assume that the solution is approximated by a linearized one-point collocation method as in Foster and Khumalo [4]. Our objective is to determine, for each $f$ under consideration, whether the numerical scheme has the same dynamical behaviour as the differential equation. In particular, we will consider cases in which the ODE has a unique, asymptotically stable periodic solution and establish conditions under which the numerical methods have the same dynamics. These special cases will take the following form:

(i) $f$ linear: $f(x, t)=a(t) x+b(t)$, where $a(t)$ and $b(t)$ are $C^{1} T$-periodic functions of $t$,

(ii) $f$ nonlinear: $f=a(t) g(x)+b(t)$, where $g(x)$ is a $C^{2}$ nonlinear function of $x$.

The linearized one-point collocation methods for the scalar nonautonomous equation (1) are given by

$$
\begin{aligned}
x_{n+1}= & \left(x_{n}+h f\left(t_{n}, x_{n}\right)+c_{1} h^{2}(\partial f / \partial t)\left(t_{n}, x_{n}\right)\right. \\
& \left.-c_{1} h x_{n}(\partial f / \partial x)\left(t_{n}, x_{n}\right)\right) \\
& \times\left(1-c_{1} h(\partial f / \partial x)\left(t_{n}, x_{n}\right)\right)^{-1},
\end{aligned}
$$

where $0 \leq c_{1} \leq 1$.

For a discussion of collocation methods in general, see Hairer et al. [5].

We begin with a description of the dynamical systems theory approach, which will be used in determining the conditions under which the methods have the same dynamical 
behaviour as the differential equations. Upon establishing these conditions, we compare them with those imposed by nonautonomous stability theory.

1.1. Dynamical Systems Approach. In what follows, we will use a technique known as stroboscopic sampling to reduce the problem of determining existence and stability of periodic solutions to existence and stability of fixed points.

Let $x_{n+1}=p\left(x_{n} ; n ; h\right)$ be the discrete system representing the numerical method, applied with fixed stepsize, to the nonautonomous differential equation.

Step 1. Using inductive arguments, write the method in the form $x_{n+1}=\phi\left(x_{0} ; n ; h\right)$.

Step 2. Choose $h$ such that the period $T=h k$. Then, $x_{k}=$ $\phi\left(x_{0} ; k ; h\right)$, and then we establish the new discrete system $X_{n+1}=\phi\left(X_{n} ; k ; h\right)$. This is known as stroboscopic sampling.

Step 3. The fixed points of the last system correspond to $T$ periodic solutions of the method. These are determined with their stability types.

The above procedure is analogous to the Poincare map of (1). Let $\Phi\left(t, x_{0}\right)$ be the $T$-periodic solution of (1), with the starting value $x(0)=x_{0}$. Then, the Poincaré map of (1) is the scalar mapping

$$
\Pi: \mathbb{R} \longrightarrow \mathbb{R} ; \quad x \longmapsto \Phi(T, x) .
$$

\section{Linear Case}

Suppose that $f=a(t) x+b(t)$, where $a$ and $b$ are $T$-periodic functions of $t$. Then, the linear nonautonomous differential equation (1) becomes

$$
x^{\prime}=a(t) x+b(t), \quad \text { for } t \geq 0, x(0)=x_{0} .
$$

If $v_{1}=\int_{0}^{T} a(t) d t<0$, then (4) has a unique $T$-periodic asymptotically stable solution. If $a(t)=0$, then (4) has a unique $T$-periodic solution that is asymptotically stable if $v_{2}=\int_{0}^{T} b(s) d s=0$ (Hale and Koçak, [3]).

Now, the linearized one-point collocation methods are given by

$$
\begin{aligned}
x_{n+1}=x_{n}+\frac{h}{1-h c_{1} a(n h)}\{ & a(n h) x_{n}+b(n h) \\
& \left.+h c_{1}\left[a^{\prime}(n h) x_{n}+b^{\prime}(n h)\right]\right\} .
\end{aligned}
$$

\subsection{Linear Case with $a(t)=0$}

Theorem 1. Suppose that a linearized one-point collocation method is used to solve the linear nonautonomous differential equation (4) with $a(t)=0$. The method tends (as $n \rightarrow \infty$, $h>0$ fixed) to a periodic solution for any starting value if and only if

$$
\sum_{r=0}^{k-1} \widetilde{b}_{r}=0
$$

where $b_{r}=b(r h)+h c_{1} b^{\prime}(r h)$ for each $r$.

Proof. Assume that $v_{2}=\int_{0}^{T} b(s) d s=0$. Taking $a(t)=0$ in (5), we obtain

$$
x_{n+1}=x_{n}+h\left\{b(n h)+h c_{1} b^{\prime}(n h)\right\} .
$$

We define

$$
\widetilde{b}_{i}=b(i h)+h c_{1} b^{\prime}(i h) \quad \text { for } i=0,1,2, \ldots
$$

and denote

$$
\Pi\left(x_{n}\right)=x_{n}+h\left\{b(n h)+h c_{1} b^{\prime}(n h)\right\},
$$

so that for a given value of $x_{0}$,

$$
x_{n+1}=x_{0}+h \sum_{r=0}^{n} \widetilde{b}_{r} .
$$

Fix $k \in \mathbb{N}$ such that $T=h k$. Consider the $k$ th iterate of $x_{0}$ under $\Pi$ :

$$
x_{k}=\Pi^{k}\left(x_{0}\right)=x_{0}+\frac{T}{k} \sum_{r=0}^{k-1} \widetilde{b}_{r}
$$

and the related iteration, which corresponds to stroboscopic sampling

$$
X_{n+1}=X_{n}+\frac{T}{k} \sum_{r=0}^{k-1} \widetilde{b}_{r},
$$

where $X_{0}=x_{0}$. If the summation on the right-hand side of (12) is zero, then the discrete system is fixed at $X_{0}$ for all $n$, which corresponds to a periodic solution. If it is nonzero, then the stroboscopic iteration has no fixed point and diverges.

Remark 2. The second term on the right-hand side of (12) can be viewed as an application of the left rectangular quadrature rule to the integral

$$
\int_{0}^{T}\left(b(s)+c_{1} \frac{T}{k} b^{\prime}(s)\right) d s,
$$

and (12) can be written as the simple map

$$
X_{n+1}=X_{n}+c,
$$

where

$$
|c|=\frac{T}{k}\left|\sum_{r=0}^{k-1} \widetilde{b}_{r}\right| .
$$




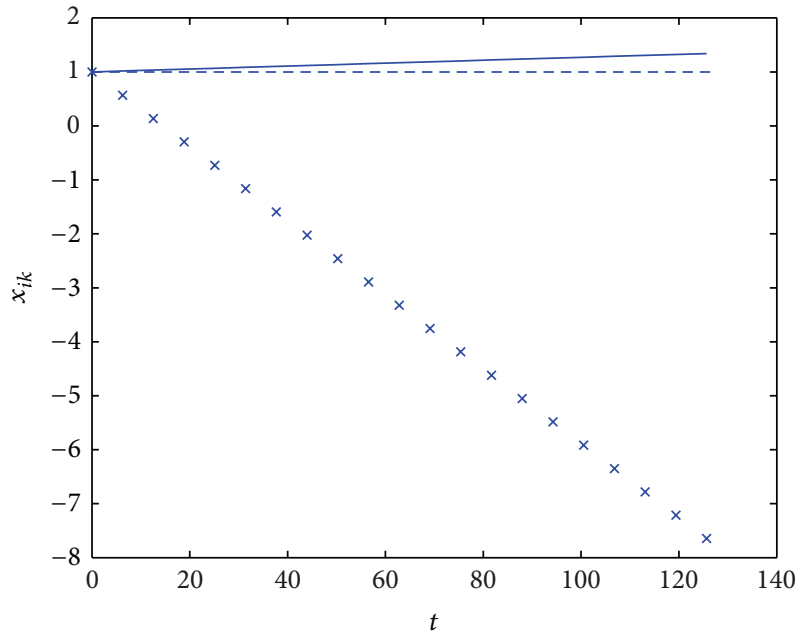

FIGURE 1: Numerical results for (12) with $c_{1}=1 / 2 . k=4(\times \times)$, and $k=5(-), k=10(---)$.

The above theorem can then be restated as follows. Suppose that a linearized one-point collocation method is used to solve the linear nonautonomous differential equation (4) with $a(t)=0$. The method tends (as $n \rightarrow \infty, h>0$ fixed) to a periodic solution for any starting value if and only if the rectangular quadrature rule, used to approximate the integral in (13), gives a zero.

Illustration. We examine the stroboscopic sampling of the numerical solution of the differential equation $x^{\prime}=\cos t e^{\sin t}$, $x(0)=1$. Figure 1 shows the numerical results for $k=4,5$, and 10 with $c_{1}=0.5$. For $k=4$, the method diverges quicker from the periodic solution than for $k=5$. For $k=10$, the divergence is negligible.

2.2. Linear Case with $a(t)=-1$. If $a(t)$ is a negative constant, (4) could be scaled in such a way that $a(t)=-1$. Then, clearly, $v_{1}=\int_{0}^{T} a(s) d s<0$, and the differential equation has a unique, asymptotically stable periodic solution.

Theorem 3. Suppose that a linearized one-point collocation method is used to solve the linear nonautonomous differential equation (4) with $a(t)=-1$. Then, for fixed $c_{1}$ and $k$, the method admits a unique periodic solution that is asymptotically stable, provided

$$
k>\frac{T}{2}\left(1-2 c_{1}\right) .
$$

Proof. If $a(t)=-1,(5)$ simplifies to

$$
x_{n+1}=x_{n}+\frac{T}{k+T c_{1}}\left[-x_{n}+b\left(\frac{n T}{k}\right)+\frac{T c_{1}}{k} b^{\prime}\left(\frac{n T}{k}\right)\right],
$$

or,

$$
\begin{aligned}
x_{n+1}= & x_{n}\left(\frac{k+T\left(c_{1}-1\right)}{k+T c_{1}}\right) \\
& +\frac{T}{k+T c_{1}}\left[b\left(\frac{n T}{k}\right)+\frac{T c_{1}}{k} b^{\prime}\left(\frac{n T}{k}\right)\right] .
\end{aligned}
$$

Proceeding in a manner analogous to the above, we can show by induction that

$$
\begin{aligned}
x_{n+1}= & x_{0}\left(\frac{k+T\left(c_{1}-1\right)}{k+T c_{1}}\right)^{n+1} \\
& +\frac{T}{k+T c_{1}} \sum_{r=0}^{n}\left(\frac{k+T\left(c_{1}-1\right)}{k+T c_{1}}\right)^{r} \widetilde{b}_{n-r},
\end{aligned}
$$

where each $\widetilde{b}_{i}$ is defined by (8).

Denoting the right-hand side of (19) by $\Pi\left(x_{n}\right)$, we can perform stroboscopic sampling and consider

$$
\begin{aligned}
x_{k}= & \Pi^{k}\left(x_{0}\right)=x_{0}\left(\frac{k+T\left(c_{1}-1\right)}{k+T c_{1}}\right)^{k} \\
& +\frac{T}{k+T c_{1}} \sum_{r=0}^{k-1}\left(\frac{k+T\left(c_{1}-1\right)}{k+T c_{1}}\right)^{r} \widetilde{b}_{k-r-1}
\end{aligned}
$$

and the associated map

$$
\begin{aligned}
X_{n+1}= & X_{n}\left(\frac{k+T\left(c_{1}-1\right)}{k+T c_{1}}\right)^{k} \\
& +\frac{T}{k+T c_{1}} \sum_{r=0}^{k-1}\left(\frac{k+T\left(c_{1}-1\right)}{k+T c_{1}}\right)^{r} \widetilde{b}_{k-r-1} .
\end{aligned}
$$

Equation (21) is just the linear map

$$
X_{n+1}=c X_{n}+d
$$

with

$$
\begin{gathered}
c=\left(\frac{k+T\left(c_{1}-1\right)}{k+T c_{1}}\right)^{k}, \\
d=\frac{T}{k+T c_{1}} \sum_{r=0}^{k-1}\left(\frac{k+T\left(c_{1}-1\right)}{k+T c_{1}}\right)^{r} \widetilde{b}_{k-r-1} .
\end{gathered}
$$

The map has a single fixed point,

$$
X^{*}=\frac{d}{1-c},
$$

which is asymptotically stable if and only if $|c|<1$; that is,

$$
k>\frac{T\left(1-2 c_{1}\right)}{2},
$$

and the result is established. 
The following results are simple consequences of the above theorem.

Corollary 4. The linearized one-point collocation method with any $c_{1} \in[1 / 2,1]$ applied to the linear nonautonomous differential equation with $a(t)=-1$ admits a unique periodic solution that is asymptotically stable for all $k>0$.

Corollary 5. The explicit Euler method for the linear nonautonomous differential equation with $a(t)=-1$ admits a unique, asymptotically stable periodic solution if and only if $k>T / 2$.

We now attempt to bound $\left|X^{*}\right|$. The following lemma gives a bound on the solution $\Phi(t)$ of (4).

Lemma 6. Let $b(t)$ be a $C^{1} T$-periodic function of $t$, and assume that (4) with $a(t)=-1$ has a unique T-periodic solution. There exist a number $M>0$ such that $|b(t)|,\left|b^{\prime}(t)\right| \leq$ $M$ and the T-periodic solution, $\Phi(t)$, satisfies

$$
|\Phi(t)| \leq M
$$

fort $\rightarrow \infty$.

Proof. The boundedness of $b(t)$ and $b^{\prime}(t)$ follows from the periodicity and continuity of both functions.

Let $\phi(t)$ be a periodic solution of (4) with $a(t)=-1$. Then, $\phi(t)$ satisfies the inequality

$$
-\phi-M \leq \phi^{\prime} \leq-\phi+M \quad \forall t .
$$

This implies that

$$
e^{-t}\left(x_{0}+M\right)-M \leq \phi \leq e^{-t}\left(x_{0}-M\right)+M ;
$$

see [3]. Thus, $\phi(t)$ is bounded for $t \geq 0$; therefore, it approaches a $T$-periodic solution $\Phi(t)$. Taking $t \rightarrow \infty$ in (28) establishes the lemma.

From the above lemma, we have $|\widehat{x}| \leq M$. Then, we have the following theorem.

Theorem 7. Let $X^{*}$, given by (24), denote the fixed point generated by the stroboscopic sampling of the numerical solution of the linear nonautonomous differential equation with $a(t)=-1$ by a linearized one-point collocation method. Then, the inequality

$$
\left|X^{*}\right| \leq M\left(1+\frac{T c_{1}}{k}\right)
$$

holds.

Proof. Since $b(t)$ is continuous, there is a number $M$ such that $|b(t)|,\left|b^{\prime}(t)\right| \leq M$ for all $t \in \mathbb{R}$. Then, for large $t$, the solution $|\Phi(t)|<M$, and hence $|\widehat{x}|<M$.
Observe that

$$
\begin{gathered}
\left|\widetilde{b}_{r}\right| \leq M_{1}=M\left(1+c_{1} \frac{T}{k}\right), \\
|d| \leq \frac{T}{k+T c_{1}} \cdot M_{1} \cdot \sum_{r=0}^{k-1}\left(\frac{k+T\left(c_{1}-1\right)}{k+T c_{1}}\right)^{r} \\
=\frac{T}{k+T c_{1}} \cdot M_{1} \\
\cdot\left\{1+\frac{k+T\left(c_{1}-1\right)}{T}\left[1-\left(\frac{k+T\left(c_{1}-1\right)}{k+T c_{1}}\right)^{k-1}\right]\right\} \\
=M_{1} \cdot\left[1-\left(\frac{k+T\left(c_{1}-1\right)}{k+T c_{1}}\right)^{k}\right] \\
=M_{1}(1-c) .
\end{gathered}
$$

Therefore,

$$
\left|X^{*}\right| \leq \frac{M_{1}(1-c)}{1-c}=M_{1}=M\left(1+\frac{T c_{1}}{k}\right) .
$$

Hence, $X^{*}$ has essentially the same bound as the periodic solution.

Example 8. Consider the linear nonautonomous equation

$$
x^{\prime}=-x+\sin t, \quad x(0)=0,
$$

which has solution $x(t)=(1 / 2)\left[\sin t-\cos t+e^{-t}\right]$. Figure 2 shows the numerical results (stroboscopic sampling) of the linearized implicit midpoint method $\left(c_{1}=1 / 2\right)$ with $k=3$ and $k=10$. Figure 3 shows the results of the explicit Euler method $\left(c_{1}=0\right)$ with $k=3$ and $k=10$. In these experiments, $T=2 \pi$; hence, the convergence to a unique periodic solution is expected for $k>\pi\left(1-2 c_{1}\right)$.

$$
\begin{aligned}
\text { 2.3. Linear Case with } a(t) & =-1+\epsilon \rho(t) \text {. Let } \\
\qquad a(t) & =-1+\epsilon \rho(t),
\end{aligned}
$$

where $\epsilon \geq 0$ is a constant and $\rho(t)$ is a $T$-periodic function of $t$. We assume that $-T+\epsilon \int_{0}^{T} \rho(s) d s<0$ so that (4) has a unique asymptotically stable periodic solution.

2.3.1. Linearized One-Point Collocation Methods. We establish conditions under which a one-point collocation method with fixed $c_{1}$ and step-size $h>0$ exhibits the same dynamical behaviour as the nonautonomous linear differential equation with $a(t)=-1+\epsilon \rho(t)$.

Notation. In what follows, we will denote $b_{n}=b(n h), b_{n}^{\prime}=$ $b^{\prime}(n h), \rho_{n}=\rho(n h)$, and $\rho_{n}^{\prime}=\rho(n h)$. Here, as before, $T=h k$ $(k \in \mathbb{N})$. 


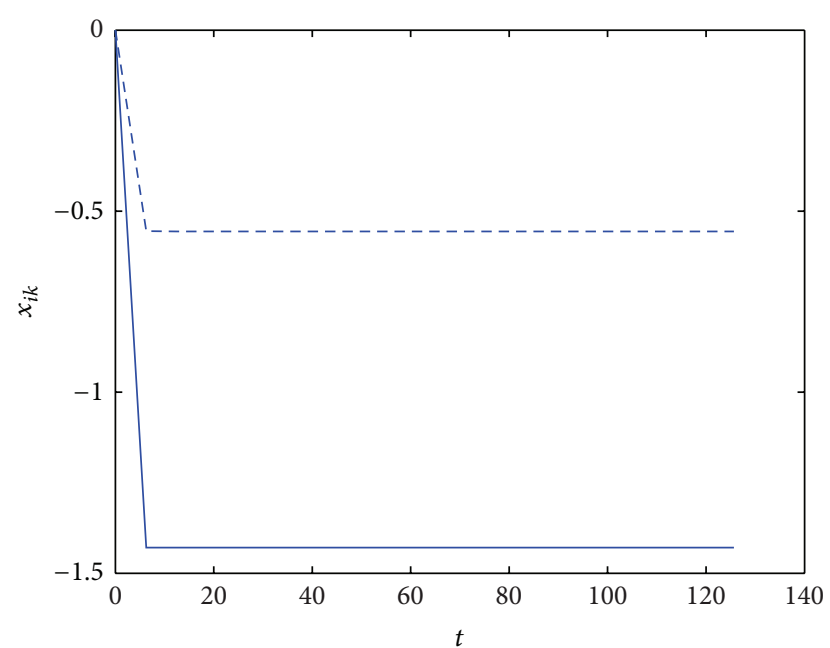

FIGURE 2: Numerical results of (32) using linearized implicit midpoint method: $k=3(-)$ and $k=10(---)$.

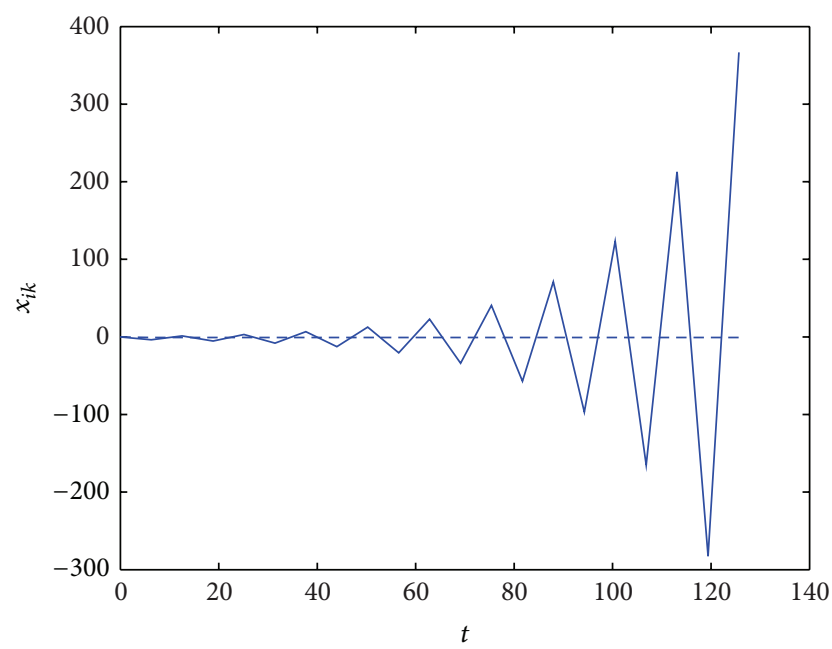

FIGURE 3: Numerical results of (32) using the explicit Euler method: $k=3(-)$ and $k=10(---)$.

Theorem 9. Suppose that a linearized one-point collocation method is used to solve a linear nonautonomous differential equation with $a(t)=-1+\epsilon \rho(t)$. Then, for fixed $c_{1}$ and $k$, the method will admit a unique periodic solution that is asymptotically stable, provided

$$
\left|\prod_{i=0}^{k-1}\left[1+\frac{k T\left(-1+\epsilon \rho_{i}\right)+c_{1} T^{2} \epsilon \rho_{i}^{\prime}}{k^{2}-k T c_{1}\left(-1+\epsilon \rho_{i}\right)}\right]\right|<1 .
$$

Proof. From (5), we deduce that the linearized collocation methods, applied to the nonautonomous linear ODE with $a(t)$ given by (33), are

$$
\begin{aligned}
x_{n+1}= & {\left[1+\frac{h\left(-1+\epsilon \rho_{n}\right)+c_{1} h^{2} \epsilon \rho_{n}^{\prime}}{1-h c_{1}\left(-1+\epsilon \rho_{n}\right)}\right] x_{n} } \\
& +\frac{h}{1-h c_{1}\left(-1+\epsilon \rho_{n}\right)} \cdot\left[b_{n}+c_{1} h b_{n}^{\prime}\right] .
\end{aligned}
$$

We can write the above as

$$
x_{n+1}=T_{n} x_{n}+H_{n} \widetilde{b}_{n}:=\Pi\left(x_{n}\right),
$$

where

$$
\begin{gathered}
T_{n}=1+\frac{h\left(-1+\epsilon \rho_{n}\right)+c_{1} h^{2} \epsilon \rho_{n}^{\prime}}{1-h c_{1}\left(-1+\epsilon \rho_{n}\right)}, \\
H_{n}=\frac{h}{1-h c_{1}\left(-1+\epsilon \rho_{n}\right)}, \\
\widetilde{b}_{n}=b_{n}+c_{1} h b_{n}^{\prime} .
\end{gathered}
$$

Proceeding by induction, we establish that

$$
x_{n+1}=x_{0} \prod_{i=0}^{n} T_{i}+\sum_{i=0}^{n} H_{i} \widetilde{b}_{i} \prod_{j=i+1}^{n} T_{j}
$$

from which we deduce that

$$
x_{k}=x_{0} \prod_{i=0}^{k-1} T_{i}+\sum_{i=0}^{k-1} H_{i} \widetilde{b}_{i} \prod_{j=i+1}^{k-1} T_{j}:=\Pi^{k}\left(x_{0}\right) .
$$

The discrete system that corresponds to stroboscopic sampling is the linear system

$$
X_{n+1}=c(\epsilon) X_{n}+d(\epsilon),
$$

where

$$
\begin{gathered}
c(\epsilon)=\prod_{i=0}^{k-1} T_{i}, \\
d(\epsilon)=\sum_{i=0}^{k-1} H_{i} \widetilde{b}_{i} \prod_{j=i+1}^{k-1} T_{j} .
\end{gathered}
$$

This system has a unique fixed point, $X^{*}$, given by (24). It is asymptotically stable if and only if $|c(\epsilon)|<1$; that is, $\left|\prod_{i=0}^{k-1} T_{i}\right|<1$, or

$$
\left|\prod_{i=0}^{k-1}\left[1+\frac{h\left(-1+\epsilon \rho_{i}\right)+c_{1} h^{2} \epsilon \rho_{i}^{\prime}}{1-h c_{1}\left(-1+\epsilon \rho_{i}\right)}\right]\right|<1 .
$$

Substituting $h=T / k$ gives the result.

2.3.2. Examples. For each of the three special values of $c_{1}$, $\rho(t)=\sin t$, and the increasing values of $\epsilon$, we determined, using (42), the minimum value of $k$ such that each method has dynamical behaviour that is the same as that of the differential equation. The results are illustrated in Figure 4.

For $\epsilon<2$, the explicit Euler method is the most restrictive of the three (i.e., comparatively larger minimum values of $k$ must be used to obtain dynamical behaviour that is the same as that of the differential equation). However, as $\epsilon$ is increased, the Explicit Euler method outperforms the linearized implicit Euler method by becoming less restrictive than that method for $\epsilon \geq 3$. For $\epsilon \geq 3$, the explicit Euler and linearized midpoint 


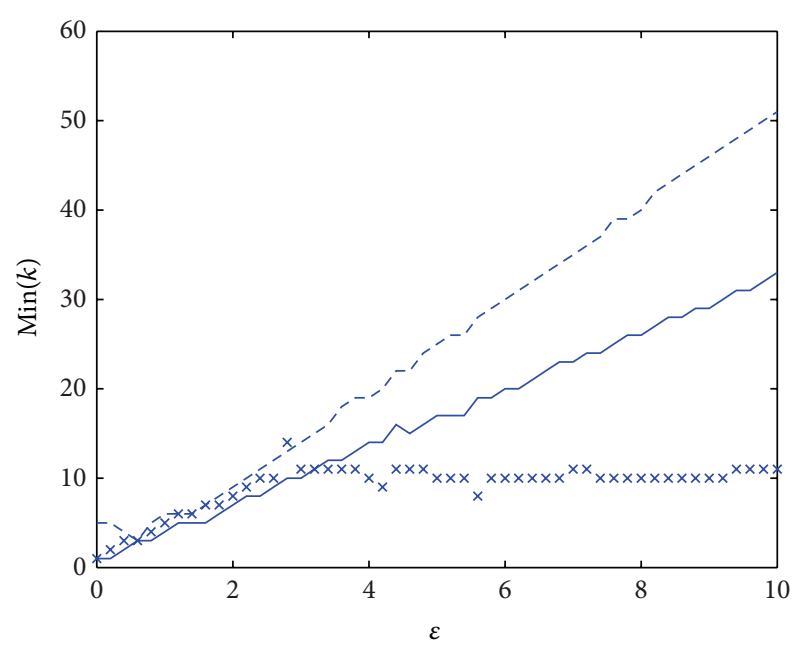

FIgURE 4: Least $k$ for unique periodic solution. The explicit Euler $(---)$, linearized implicit midpt $(-)$, and the linearized implicit Euler $(\times \times)$.

methods give comparable results, and for those values of $\epsilon$, the linearized implicit Euler method becomes more and more restrictive in comparison to the other two.

Finally, we develop a bound on $\left|X^{*}\right|$. For the comparison purposes, we present the following lemma, which can be proved in a manner analogous to Lemma 6.

Lemma 10. Let $b(t), \rho(t)$ be $C^{1} T$-periodic functions oft. There exist numbers $M, M_{2}>0$ such that $|b(t)|,\left|b^{\prime}(t)\right| \leq M,|\rho(t)|$, $\left|\rho^{\prime}(t)\right| \leq M_{2}$, and the solution $\Phi(t)$ satisfies

$$
|\Phi(t)| \leq \frac{M}{\left|1-\epsilon M_{2}\right|}
$$

ast $\rightarrow \infty$.

From the lemma, we have $|\widehat{x}| \leq M /\left|1-\epsilon M_{2}\right|$.

Theorem 11. Let $X^{*}$, given by (24), be the fixed point generated by the stroboscopic sampling of the numerical solution of the linear nonautonomous differential equation with $a(t)=-1+$ $\epsilon \rho(t)$ by a linearized collocation method. Then, the following inequality holds:

$$
\left|X^{*}\right| \leq \frac{M\left(1+T c_{1} / k\right)}{\left|1-h c_{1} \epsilon M_{2}-\epsilon M_{2}\right|} .
$$

Proof. Since $b(t)$ and $\rho(t)$ are $C^{1}$ and periodic, there are numbers $M, M_{2}$ such that $|b(t)|,\left|b^{\prime}(t)\right| \leq M$ and $|\rho(t)|$, $\left|\rho^{\prime}(t)\right| \leq M_{2}$ for all $t \in \mathbb{R}$.

For each $i$,

$$
\begin{gathered}
\left|T_{i}\right| \leq\left|\frac{1+h c_{1}+h \epsilon M_{2}-h}{1+h c_{1}-h c_{1} \epsilon M_{2}}\right|, \\
\left|H_{i}\right| \leq \frac{h}{\left|1+h c_{1}-h c_{1} \epsilon M_{2}\right|}, \\
\left|\widetilde{b}_{i}\right| \leq M_{1}=M\left(1+c_{1} h\right),
\end{gathered}
$$

where $h=T / k$. If

$$
v(\epsilon)=\frac{1+h c_{1}-h c_{1} \epsilon M_{2}+h \epsilon M_{2}+h^{2} c_{1} \epsilon M_{2}-h}{1+h c_{1}-h c_{1} \epsilon M_{2}},
$$

then

$$
\begin{aligned}
|d(\epsilon)| \leq & \frac{h}{\left|1+h c_{1}-h c_{1} \epsilon M_{2}\right|} \cdot M_{1} \cdot\left|\sum_{i=0}^{k-1} v^{k-i-1}\right| \\
= & \frac{h M_{1}}{\left|1+h c_{1}-h c_{1} \epsilon M_{2}\right|} \cdot\left|\frac{1-v^{k}}{h\left(1-c_{1} h \epsilon M_{2}-\epsilon M_{2}\right)}\right| \\
& \cdot\left|1+h c_{1}-h c_{1} \epsilon M_{2}\right| \\
= & M_{1} \cdot\left|\frac{1-v^{k}}{\left(1-c_{1} h \epsilon M_{2}-\epsilon M_{2}\right)}\right| .
\end{aligned}
$$

On the other hand, we deduce from the definition of $c(\epsilon)$ that

$$
\begin{aligned}
& |1-c(\epsilon)| \\
& \quad \geq\left|1-\left(\frac{1+h c_{1}-h+h \epsilon M_{2}-h c_{1} \epsilon M_{2}+h^{2} c_{1} \epsilon M_{2}}{1+h c_{1}-h c_{1} \epsilon M_{2}}\right)^{k}\right| .
\end{aligned}
$$

Hence,

$$
\left|X^{*}(\epsilon)\right| \leq \frac{M_{1}}{\left|1-c_{1} h \epsilon M_{2}-\epsilon M_{2}\right|}=\frac{M\left(1+c_{1} h\right)}{\left|1-c_{1} h \epsilon M_{2}-\epsilon M_{2}\right|},
$$

which is identical to the inequality (44).

If we substitute $\epsilon=0$ in (49), we obtain (31) as expected. Here, as well, the bound for $X^{*}(\epsilon)$ is the same as that of the periodic solution as $h \rightarrow 0$.

We would like to obtain a relationship between the dynamical approach study and stability analysis. We introduce a natural stability criterion for the differential equation as well as any numerical method used to discretize it.

2.4. Conditional AN Stability and AN Stability. We consider the problem of determining a criterion for some sort of "controlled behaviour" of the solutions of the methods. We adopt a linear stability criterion that is based on the scalar test equation

$$
x^{\prime}=a(t) x,
$$

where $a(t) \in \mathbb{C}$. If $\operatorname{Re}(a(t))<0$ for all $t \in\left[\beta_{1}, \beta_{2}\right]$, then

$$
x(t+h)=K x(t), \quad|K| \leq 1
$$

for all $x \in\left[\beta_{1}, \beta_{2}\right]$ and $h>0$.

Definition 12. A numerical method is said to be conditionally AN stable for some $h>0$ if, when applied to the test equation (50) with $\operatorname{Re}(a(t))<0$, for all $t$,

$$
x_{n+1}=\widetilde{K}(h) x_{n}, \quad|\widetilde{K}(h)| \leq 1
$$

holds for all $n \in \mathbb{N}$. 
If this condition is satisfied for all $h>0$, then the method is AN stable.

For a discussion of AN stability, see Lambert [6] and Stuart and Humphries [7]

The following simple result gives a condition under which the linearized one-point collocation methods are conditionally AN stable.

Theorem 13. Assuming real $a(t)$, the linearized one-point collocation methods are conditionally AN stable if and only if

$$
\left(1-2 c_{1}\right) a(t)+c_{1} h a^{\prime}(t) \geq-\frac{2}{h}
$$

and $a(t)+c_{1} h a^{\prime}(t) \leq 0$.

Proof. Use the above test equation in (2). On the other hand, if (2) is not satisfied, the method fails the stability criterion and is not AN stable.

Examples:

(1) if $a(t)=-1$, then the linearized one-point collocation methods are conditionally AN stable if and only if

$$
\frac{h}{2}\left(1-2 c_{1}\right) \leq 1 .
$$

It is easy to observe that the methods are AN stable if $c_{1} \geq 1 / 2$,

(2) if $a(t)=-1+\epsilon \rho(t)$, where $\epsilon, \rho(t) \in \mathbf{C}$, and $\epsilon\left(\rho(t)+c_{1} h \rho^{\prime}(t)\right) \leq 1$, then the linearized one-point collocation methods are conditionally AN stable if and only if

$$
-1+\epsilon \rho(t)+c_{1}\left(h \epsilon \rho^{\prime}(t)+2-2 \epsilon \rho(t)\right) \geq-\frac{2}{h} .
$$

We have proved the existence of a relationship between the linear stability theory of the collocation methods and the existence and asymptotic stability of periodic solutions, identified via stroboscopic sampling. This relationship is stated in the following theorem.

Theorem 14. Suppose that a linearized one-point collocation method is used to solve a linear nonautonomous equation of the form discussed in Sections 2.2 or 2.3 which has periodic coefficients and possesses a unique, asymptotically stable periodic solution. Then, the following conditions are equivalent:

(a) the method is AN stable,

(b) the method yields the same dynamics as the differential equation.

The last theorem is very significant, since it gives us a bridge connecting standard stability theory with dynamical systems. Naturally, we would like to find out if there is a corresponding result for the nonlinear case, which we now consider.

\section{Nonlinear Case}

We consider the nonlinear equation,

$$
x^{\prime}=a(t) g(x)+b(t), \quad \text { for } t \geq 0, x(0)=x_{0},
$$

where $b(t)$ is a $T$-periodic function of $t$ and $g(x)$ is a $C^{2}$ nonlinear function of $x$.

Massera [8] proved that if a nonlinear equation of the form (56) has the uniqueness property with respect to the initial conditions, the existence of a bounded solution implies the existence of a $T$-periodic solution.

3.1. Linearized One-Point Collocation Methods. The linearized collocation methods, applied to (56), are given by

$$
x_{n+1}=x_{n}+\frac{h \widetilde{a}_{n} g\left(x_{n}\right)}{1-h a_{n} c_{1} g^{\prime}\left(x_{n}\right)} h+\frac{h \widetilde{b}_{n}}{1-h a_{n} c_{1} g^{\prime}\left(x_{n}\right)},
$$

where $\widetilde{b}_{n}=b_{n}+c_{1} h b_{n}^{\prime}$ and $\widetilde{a}_{n}=a_{n}+c_{1} h a_{n}^{\prime}$.

We perform a simplification on the third term of (57) that takes the form of evaluating the derivative of $g$ at the starting value, instead, at each step. The resulting method, that will be referred to as a simplified linearized one-point collocation method, is

$$
x_{n+1}=x_{n}+h \widetilde{a}_{n} G\left(x_{n} ; n\right)+H_{n} \widetilde{b}_{n},
$$

where

$$
\begin{gathered}
H_{n}=\frac{h}{1-h a_{n} c_{1} g^{\prime}\left(x_{0}\right)}, \\
G(x ; n)=\frac{g(x)}{1-h a_{n} c_{1} g^{\prime}(x)} .
\end{gathered}
$$

3.2. The Dynamical Systems Approach. We would like to take the dynamical systems approach and determine the conditions under which (58), applied to the differential equation (56), yields the same dynamics as the continuous system.

We rewrite (58) as

$$
h \widetilde{a}_{n} G\left(x_{n} ; n\right)=x_{n+1}-x_{n}-H_{n} \widetilde{b}_{n} .
$$

Inductively, we can show that

$$
x_{n+1}=x_{0}+\sum_{r=0}^{n} H_{r} \widetilde{b}_{r}+h \sum_{r=0}^{n} \widetilde{a}_{r} G\left(x_{r} ; r\right) .
$$

We choose an integer $k$ such that $T=h k$. Sampling stroboscopically in the iteration above, we get

$$
x_{k}=x_{0}+\sum_{r=0}^{k-1} H_{r} \widetilde{b}_{r}+h \sum_{r=0}^{k-1} \widetilde{a}_{r} G\left(x_{r} ; r\right)
$$

and associate this with the discrete system

$$
X_{n+1}=X_{n}+\sum_{r=0}^{k-1} H_{r} \widetilde{b}_{r}+h \sum_{r=0}^{k-1} \widetilde{a}_{r} G\left(\widetilde{X}_{r} ; r\right),
$$


where

$$
\begin{gathered}
\widetilde{X}_{0}=X_{n} \\
\widetilde{X}_{r}=X_{n}+\sum_{i=0}^{r-1} H_{r} \widetilde{b}_{r}+h \sum_{i=0}^{r-1} \widetilde{a}_{i} G\left(\widetilde{X}_{i} ; i\right) \quad \text { for } r=1,2, \ldots, k-1 .
\end{gathered}
$$

The fixed points of (63) correspond to periodic solutions of (56). Fixed points are points, $X^{*}$, such that

$$
\sum_{r=0}^{k-1} H_{r} \widetilde{b}_{r}+h \sum_{r=0}^{k-1} \widetilde{a}_{r} G\left(\widetilde{X}_{r} ; r\right)=0
$$

where

$$
\widetilde{X}_{r}=X^{*}+\sum_{i=0}^{r-1} H_{i} \widetilde{b}_{i}+h \sum_{i=1}^{r-1} \widetilde{a}_{i} G\left(\widetilde{X}_{i} ; i\right) \quad \text { for } r=1,2, \ldots, k-1 \text {, }
$$

and $X_{0}=X^{*}$. Define the sequence of functions $F_{1}(x)$, $F_{2}(x), \ldots$ by

$$
F_{k}(x)=\sum_{r=0}^{k-1}\left[\tilde{a}_{r} h G\left(\tilde{x}_{r} ; r\right)+H_{r} \tilde{b}_{r}\right]
$$

where $\tilde{x}_{0}=x$ and

$$
\tilde{x}_{r}=x+\sum_{i=0}^{r-1}\left[\widetilde{a}_{i} h G\left(\tilde{x}_{i} ; i\right)+H_{i} \tilde{b}_{i}\right]
$$

for $r=1,2, \ldots, k-1$.

The theorem below gives conditions under which the simplified linearized one-point collocation methods, applied to (56), exhibit dynamical behaviour that is the same as the differential equation.

Theorem 15. Assume that the differential equation (56) has a unique solution. If

(i) $g^{\prime \prime}(x) g(x) \leq 0$,

(ii) $c_{1} a^{\prime}(t)<-a(t) / h$ for all $t$,

(iii) $\operatorname{Max} G^{\prime}(x ; t) \leq 1 / h|\widetilde{a}(t)|$

(iv) $\left|F_{k}^{\prime}(x)\right|<1$ for all $k \in \mathbb{N}$, where $F_{k}(x)$ is given by (67),

then a simplified one-point collocation method has a periodic solution for any $k=T / h$; this solution is unique and asymptotically stable.
Proof. Finding possible fixed points of (63), hence periodic solutions of the methods, is the same as finding zeros of (67). This is equivalent to solving the nonlinear system:

$$
\begin{aligned}
& -h \tilde{a}_{0} G\left(x^{*} ; 0\right)-h \tilde{a}_{1} G\left(\tilde{x}_{1} ; 1\right)-h \tilde{a}_{2} G\left(\tilde{x}_{2} ; 2\right)-\cdots \\
& -h \tilde{a}_{k-1} G\left(\tilde{x}_{k-1} ; k-1\right)-H_{0} \tilde{b}_{0}-H_{1} \tilde{b}_{1}-\cdots \\
& -H_{k-1} \widetilde{b}_{k-1}=0 \text {, } \\
& \tilde{x}_{1}-x^{*}-h \widetilde{a}_{0} G\left(x^{*} ; 0\right)-H_{0} \widetilde{b}_{0}=0, \\
& \tilde{x}_{2}-x^{*}-h \widetilde{a}_{0} G\left(x^{*} ; 0\right)-h \widetilde{a}_{1} G\left(\tilde{x}_{1} ; 1\right)-H_{0} \widetilde{b}_{0}-H_{1} \widetilde{b}_{1}=0 \text {, } \\
& \tilde{x}_{k-1}-x^{*}-h \widetilde{a}_{0} G\left(x^{*} ; 0\right)-h \widetilde{a}_{1} G\left(\tilde{x}_{1} ; 1\right)-h \widetilde{a}_{2} G\left(\tilde{x}_{2} ; 2\right)-\cdots \\
& -h \tilde{a}_{k-2} G\left(\tilde{x}_{k-2} ; k-2\right)-H_{0} \tilde{b}_{0}-H_{1} \tilde{b}_{1}-\cdots \\
& -H_{k-2} \widetilde{b}_{k-2}=0 \text {. }
\end{aligned}
$$

The above system is of the form $\mathbf{F}(\mathbf{x})=\mathbf{0}$, where $\mathbf{F}$ is a nonlinear function of

$$
\mathbf{x}=\left(\begin{array}{c}
x^{*}=\tilde{x}_{0} \\
\tilde{x}_{1} \\
\tilde{x}_{2} \\
\vdots \\
\tilde{x}_{k-1}
\end{array}\right) \text {. }
$$

To prove existence, it is sufficient to show that the Jacobian matrix, $\mathrm{J}(\mathbf{F})$, of $\mathbf{F}$ is nonsingular. Now,

$$
\mathrm{J}(\mathbf{F})=\left(\begin{array}{ccccccc}
d_{0} & d_{1} & d_{2} & d_{3} & \cdots & d_{k-2} & d_{k-1} \\
-1+d_{0} & 1 & 0 & 0 & \cdots & 0 & 0 \\
-1+d_{0} & d_{1} & 1 & 0 & 0 & \cdots & 0 \\
-1+d_{0} & d_{1} & d_{2} & 1 & 0 & \cdots & 0 \\
& & & \vdots & & & \\
-1+d_{0} & d_{1} & d_{2} & \cdots & d_{k-2} & d_{k-1} & 1
\end{array}\right)
$$

where $d_{i}=-h \widetilde{a}_{i} G^{\prime}\left(\tilde{x}_{i} ; i\right)$ for each $i$. We perform one elementary row operation: row $1 \rightarrow$ row 1 -row $k$. The matrix becomes

$$
\left(\begin{array}{ccccccc}
1 & 0 & 0 & 0 & \cdots & 0 & -1+d_{k-1} \\
-1+d_{0} & 1 & 0 & 0 & \cdots & 0 & 0 \\
-1+d_{0} & d_{1} & 1 & 0 & 0 & \cdots & 0 \\
-1+d_{0} & d_{1} & d_{2} & 1 & 0 & \cdots & 0 \\
& & & \vdots & & & \\
-1+d_{0} & d_{1} & d_{2} & \cdots & d_{k-2} & d_{k-1} & 1
\end{array}\right)
$$

It is easy to see that the above matrix is nonsingular. 
To prove uniqueness, let $x_{1}$ and $x_{2}$ be two fixed points of (63). Then, from (67),

$$
\begin{aligned}
F_{k}\left(x_{1}\right)= & F_{k-1}\left(x_{1}\right)+h \widetilde{a}_{k-1} G\left(x_{1}+F_{k-1}\left(x_{1}\right) ; k-1\right) \\
& +H_{k-1} \widetilde{b}_{k-1}=0, \\
F_{k}\left(x_{2}\right)= & F_{k-1}\left(x_{2}\right)+h \tilde{a}_{k-1} G\left(x_{2}+F_{k-1}\left(x_{2}\right) ; k-1\right) \\
& +H_{k-1} \tilde{b}_{k-1}=0 .
\end{aligned}
$$

Subtracting (74) from (73) and using the mean value theorem gives

$$
\begin{aligned}
& \left(x_{1}-x_{2}\right)\left\{F_{k-1}^{\prime}\left(\alpha_{1}\right)+h \tilde{a}_{k-1} G^{\prime}\left(\alpha_{2} ; k-1\right)\left(1+F_{k-1}^{\prime}\left(\alpha_{1}\right)\right)\right\} \\
& =0
\end{aligned}
$$

which may alternatively be written as

$$
\begin{gathered}
\left(x_{1}-x_{2}\right)\left\{F_{k-1}^{\prime}\left(\alpha_{1}\right)\left(1+h \widetilde{a}_{k-1} G^{\prime}\left(\alpha_{2} ; k-1\right)\right)\right. \\
\left.+h \widetilde{a}_{k-1} G^{\prime}\left(\alpha_{2} ; k-1\right)\right\}=0 .
\end{gathered}
$$

In the above equations, $\alpha_{1}$ is between $x_{1}$ and $x_{2}$, and $\alpha_{2}$ is between $x_{1}+F_{k-1}\left(x_{1}\right)$ and $x_{2}+F_{k-1}\left(x_{2}\right)$.

From the hypotheses of the theorem, we have that $F^{\prime}<0$, $G^{\prime}>0, \tilde{a}_{k-1}<0$, and $1 \geq 1+h \widetilde{a}_{k-1} G^{\prime}\left(\alpha_{2} ; k-1\right) \geq 0$. Thus, $x_{1}=x_{2}$.

The periodic solution is asymptotically stable since $\mid 1+$ $F^{\prime} \mid<1$. This completes the proof.

3.3. Numerical Experiments. Consider the nonlinear nonautonomous ODE

$$
x^{\prime}(t)=-x^{3}+\sin t, \quad x(0)=1 .
$$

The numerical results (stroboscopic sampling) of each of the three cases are given in Figures 5, 6, and 7.

The methods give comparable results, but the implicit midpoint converges faster to the periodic solutions than the other two.

3.4. Nonlinear Stability Theory. We wish to establish conditions under which numerical methods for the solution of (56) behave in a "controlled" manner. We will view such controlled behaviour in the system meaning that neighbouring solution curves get closer and closer together as $t$ increases. This concept, called contractivity, is discussed, for instance, in Lambert [6]. As in the linear case, we will contrast the conditions for stability with the existence and uniqueness of a periodic solution in the numerical methods.

We briefly state the concepts of contractivity and conditional BN stability.

Definition 16. Let $x(t)$ and $\bar{x}(t)$ be any two solutions of the differential equation $x^{\prime}=f(x, t)$, satisfying initial conditions $x(0)=\eta, \bar{x}(0)=\bar{\eta}, \eta \neq \bar{\eta}$. Then, if

$$
\left\|x\left(t_{2}\right)-\bar{x}\left(t_{2}\right)\right\| \leq\left\|x\left(t_{1}\right)-\bar{x}\left(t_{1}\right)\right\|
$$

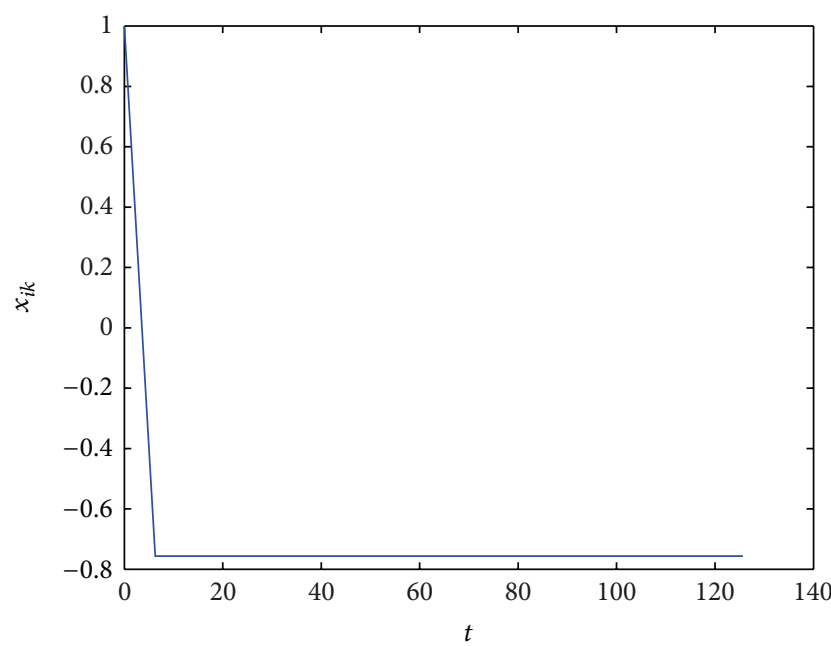

FIGURE 5: Numerical results for (77) with $c_{1}=0 . k=20$.

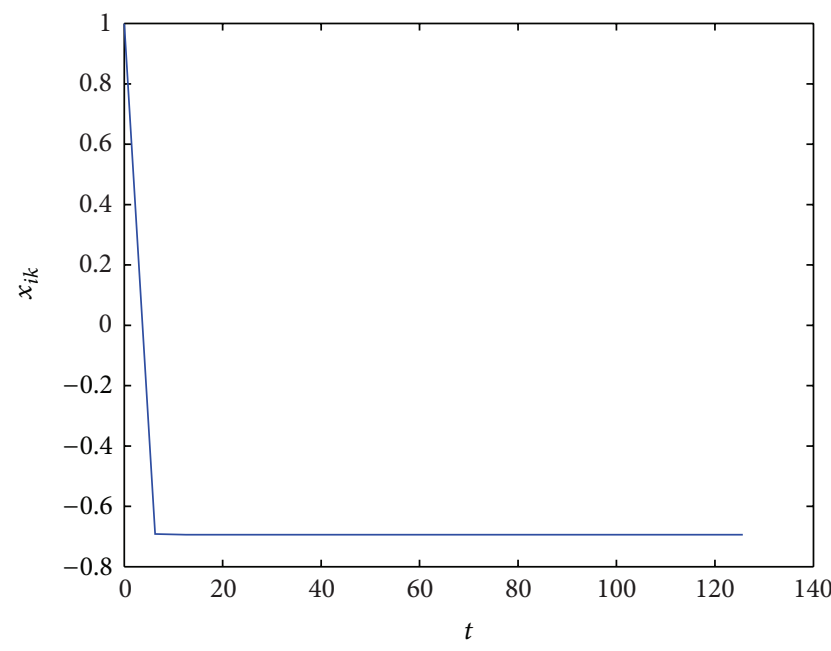

Figure 6: Numerical results for (77) with $c_{1}=1 / 2 . k=20$.

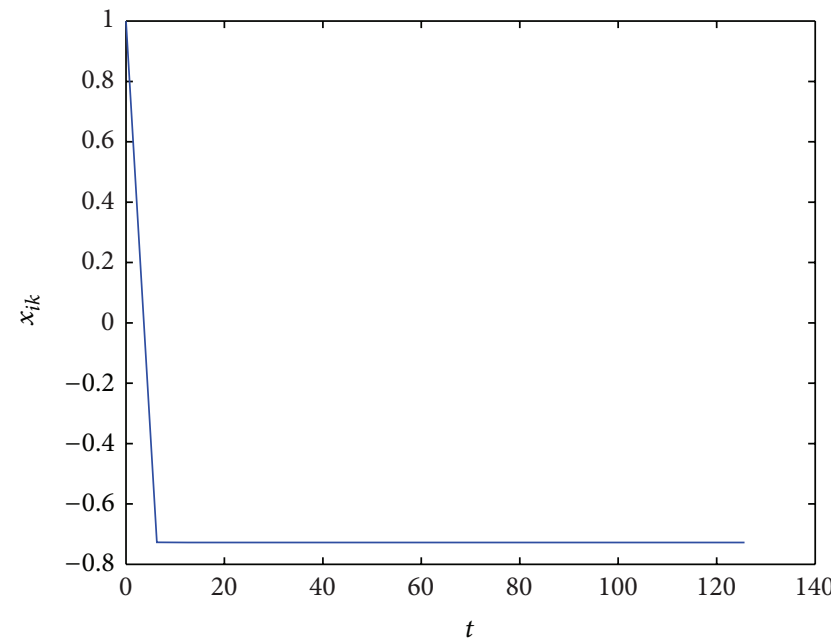

FIgURE 7: Numerical results for (77) with $c_{1}=1 . k=20$. 
holds under the $\mathbb{R}^{m}$ norm $\|\cdot\|$ for all $t_{1}, t_{2}$ such that $\beta_{1} \leq t_{1} \leq$ $t_{2} \leq \beta_{2}$, the solutions of the system are said to be contractive in $\left[\beta_{1}, \beta_{2}\right]$.

The discrete analog of the above definition is given below.

Definition 17. Let $\left\{x_{n}\right\}$ and $\left\{\bar{x}_{n}\right\}$ be two numerical solutions generated by a numerical method with different starting values. Then, if

$$
\left\|x_{n+1}-\bar{x}_{n+1}\right\| \leq\left\|x_{n}-\bar{x}_{n}\right\|, \quad 0 \leq n \leq N,
$$

the numerical solutions are said to be contractive for $n \in$ $[0, N]$.

Definition 18. The system $x^{\prime}=f(x, t)$ is dissipative in $\left[\beta_{1}, \beta_{2}\right]$ if

$$
\langle f(x, t)-f(\bar{x}, t), x-\bar{x}\rangle \leq 0
$$

holds for all $x, \bar{x} \in \mathbb{R}$ and for all $t \in\left[\beta_{1}, \beta_{2}\right]$.

It is easy to show that the solutions of a dissipative system are contractive under the norm induced by the inner product in (80). It is desirable that a numerical method, used with fixed stepsize $h>0$ to solve a dissipative system, gives contractive solutions. This brings us to the concepts of conditional BN stability, BN stability and nonlinear nonautonomous stability criteria.

Definition 19. If a numerical method, applied with fixed steplength $h>0$ to (56) satisfying (80), generates contractive solutions, the method is said to be conditionally BN stable. If the method generates contractive solutions when applied with any $h>0$, then it it is BN stable (Lambert [6]).

The concepts of AN and BN stability are equivalent for the nonconfluent Runge-Kutta methods.

To determine the conditional BN stability of the methods, we use the scalar test system

$$
x^{\prime}=a(t) g(x)
$$

where, as before, $a(t) \in \mathbb{C}$ and $g(x) \in C^{1}$. This system is dissipative if

$$
a(t)\langle g(x)-g(\bar{x}), x-\bar{x}\rangle=a(t) g^{\prime}(\xi)|x-\bar{x}|^{2} \leq 0,
$$

where $\xi$ lies between $x$ and $\bar{x}$ and $\langle\cdot, \cdot\rangle$ is an inner product in $\mathbb{R}$. The condition is satisfied if $a(t) \leq 0$ for all $t$ and $g^{\prime}(x) \geq 0$ for all $x$. Therefore, the existence and uniqueness of a periodic solution in (56) is a sufficient condition for the dissipativity of the system.

Now, we determine the conditions under which the linearized one-point collocation methods are conditionally BN stable. Applying the methods to the test system (81) for two different initial conditions gives

$$
\begin{aligned}
& x_{n+1}=x_{n}+h \widetilde{a}_{n} G\left(x_{n} ; n\right), \\
& \bar{x}_{n+1}=\bar{x}_{n}+h \tilde{a}_{n} G\left(\bar{x}_{n} ; n\right) .
\end{aligned}
$$

Subtracting (84) from (83) and using the mean value theorem gives

$$
\left|x_{n+1}-\bar{x}_{n+1}\right|=\left|\left(x_{n}-\bar{x}_{n}\right)\right| \cdot\left|1+h \widetilde{a}_{n} G^{\prime}\left(\xi_{n} ; n\right)\right|,
$$

where $\xi_{n}$ lies between $x_{n}$ and $\bar{x}_{n}$.

The solutions generated by the methods are contractive if and only if $\left|1+h \widetilde{a}_{n} G^{\prime}\left(\xi_{n} ; n\right)\right| \leq 1$. Assuming $\widetilde{a}(t)<0$ for all $t$ and $G^{\prime}(x ; t) \geq 0$ for all $x$ and $t$, the methods are conditionally $\mathrm{BN}$ stable if and only if

$$
G^{\prime}(x ; t) \leq \frac{2}{h \widetilde{a}(t)} .
$$

Remark 20. If we let $g(x)=x$ (the linear case), then condition (86) collapses to (53), which is the condition for conditional AN stability.

3.5. Discussion. We have, in Theorem 15, established sufficient conditions for the simplified linearized one-point collocation methods to exhibit the same dynamics as the nonlinear nonautonomous ODE. Conditions (i) and (ii) are required for conditional BN stability as well, but condition (iii) is not necessary for the existence of a unique, asymptotically stable solution. In fact, we can come to the same conclusion as in Theorem 15 if, in (75),

$$
F^{\prime}(x)\left[1+h \widetilde{a}(t) G^{\prime}(x ; t)\right]+h \widetilde{a}(t) G^{\prime}(x ; t) \neq 0
$$

for all $x$ and $t$. The following theorem shows that condition (87) is satisfied if the method is conditionally BN stable.

Theorem 21. Suppose that a simplified linearized one-point collocation method is used to solve a nonlinear nonautonomous equation of the form (56) with periodic coefficients which has a unique, asymptotically stable periodic solution. Then, the method yields a unique periodic solution that is asymptotically stable if it is conditionally BN stable.

Proof. Assume the conditional BN stability. To prove the theorem, it is sufficient to establish condition (87). From conditional BN stability, we have $-1 \leq 1+h \widetilde{a} G^{\prime} \leq 1$. Recall that $-1 \leq F^{\prime}<0$ from condition (iv). Therefore,

$$
F^{\prime}+h \widetilde{a} G^{\prime} \leq F^{\prime}\left(1+h \widetilde{a} G^{\prime}\right)+h \widetilde{a} G^{\prime} \leq-F^{\prime}+h \widetilde{a} G^{\prime} .
$$

Since $0<-F^{\prime} \leq 1$, if $-1 \leq 1+h \tilde{a} G^{\prime}<0$,

$$
F^{\prime}\left(1+h \tilde{a} G^{\prime}\right)+h \tilde{a} G^{\prime} \leq 1+h \tilde{a} G^{\prime}<0 .
$$

If $0 \leq 1+h \widetilde{a} G^{\prime} \leq 1$,

$$
F^{\prime}+h \widetilde{a} G^{\prime} \leq F^{\prime}\left(1+h \tilde{a} G^{\prime}\right)+h \widetilde{a} G^{\prime} \leq h \widetilde{a} G^{\prime}<0 .
$$

This proves the theorem.

Remark 22. The above theorem is somewhat similar to Theorem 14 (in the linear case) if we replace the concept of conditional BN stability by conditional AN stability. 


\section{Conclusion}

We already knew that numerical methods can introduce spurious behaviours into the solution for autonomous equations. By concentrating on linear and nonlinear nonautonomous equations with unique periodic solutions and discretizing them using one-point collocation methods, we were interested in the existence of periodic solutions in the numerical methods.

We found that the results obtained from the dynamical systems approach are closely linked to those that are imposed by standard stability analysis. It has been shown that for linear and nonlinear nonautonomous differential equations of the form considered in this chapter, there is a relationship between conditional AN or BN stability of a one-point collocation method and the method yielding the same dynamical behaviour as the differential equation under consideration.

\section{References}

[1] A. Iserles, "Stability and dynamics of numerical methods for nonlinear ordinary differential equations," IMA Journal of Numerical Analysis, vol. 10, no. 1, pp. 1-30, 1990.

[2] A. Stuart, "Linear instability implies spurious periodic solutions," IMA Journal of Numerical Analysis, vol. 9, no. 4, pp. 465486, 1989.

[3] J. K. Hale and H. Koçak, Dynamics and Bifurcations, vol. 3, Springer, New York, NY, USA, 1991.

[4] A. Foster and M. Khumalo, "Transformation of local bifurcations under collocation methods," Journal of the Korean Mathematical Society, vol. 48, no. 6, pp. 1101-1123, 2011.

[5] E. Hairer, S. P. Nørsett, and G. Wanner, Solving Ordinary Differential Equations. I, vol. 8, Springer, Berlin, Germany, 2nd edition, 1993.

[6] J. D. Lambert, Numerical Methods for Ordinary Differential Systems, John Wiley \& Sons, New York, NY, USA, 1991.

[7] A. M. Stuart and A. R. Humphries, Dynamical Systems and Numerical Analysis, vol. 2, Cambridge University Press, New York, NY, USA, 1996.

[8] J. L. Massera, "The existence of periodic solutions of systems of differential equations," Duke Mathematical Journal, vol. 17, pp. 457-475, 1950. 


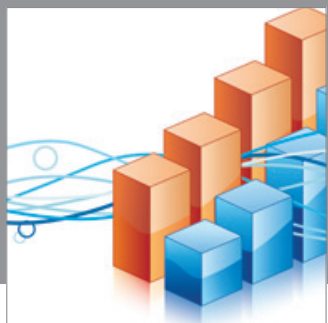

Advances in

Operations Research

mansans

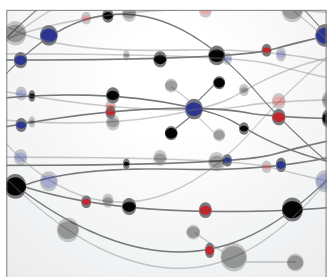

The Scientific World Journal
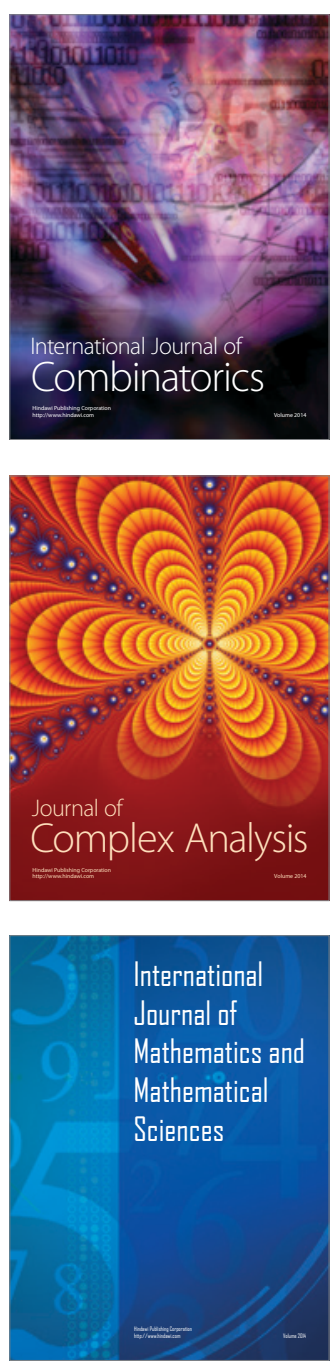
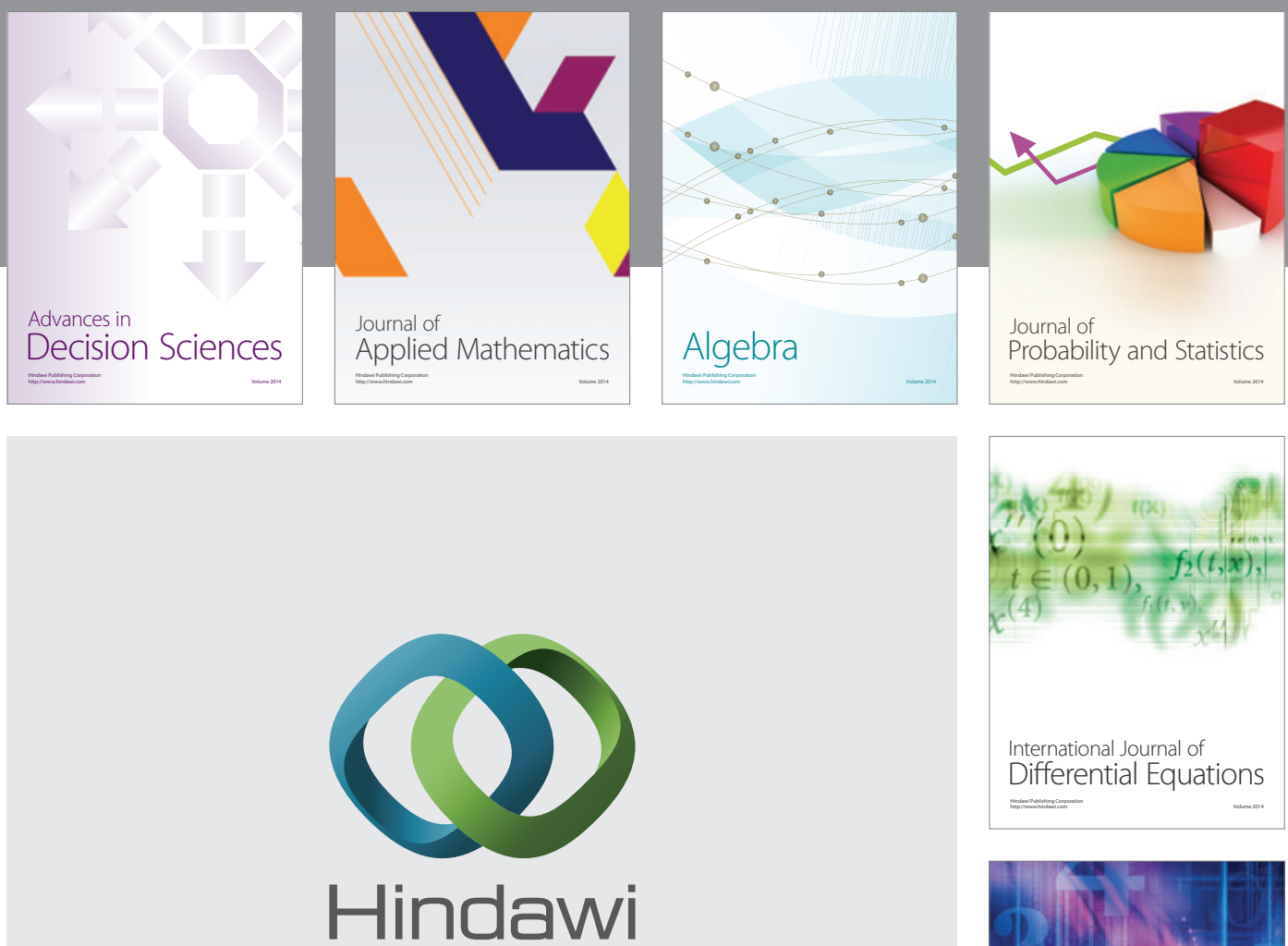

Submit your manuscripts at http://www.hindawi.com
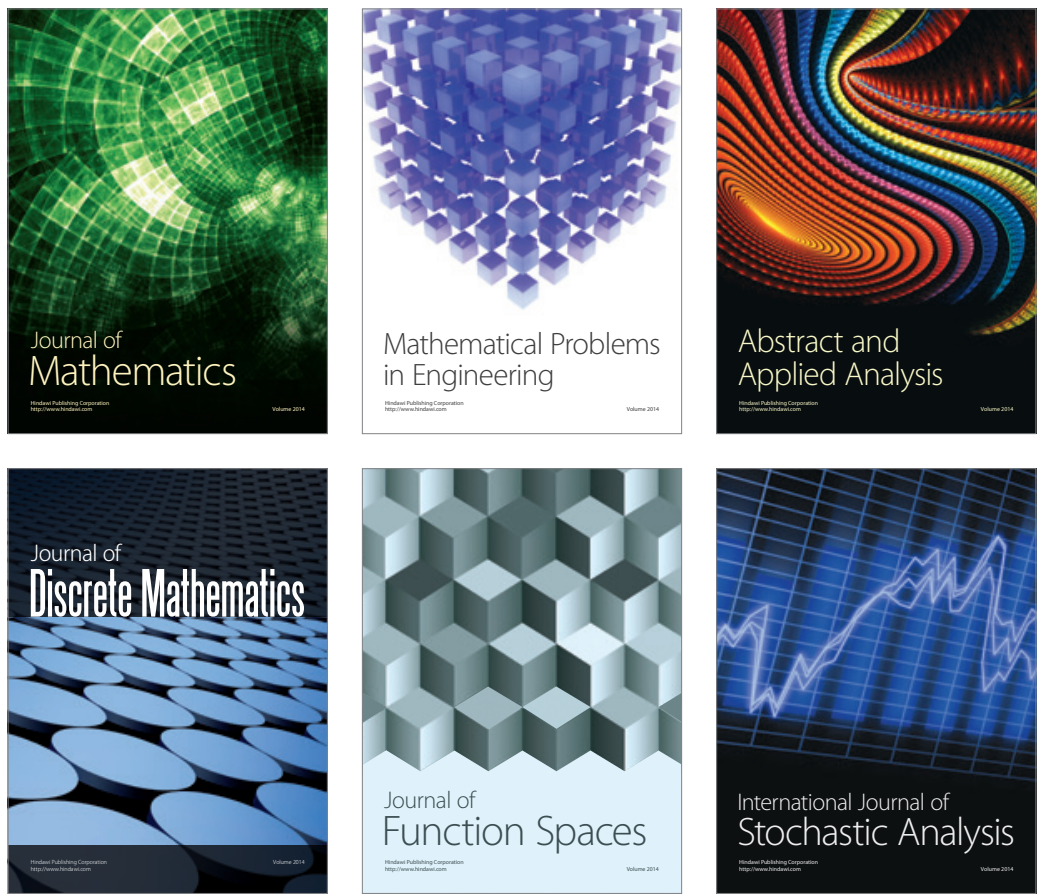

Journal of

Function Spaces

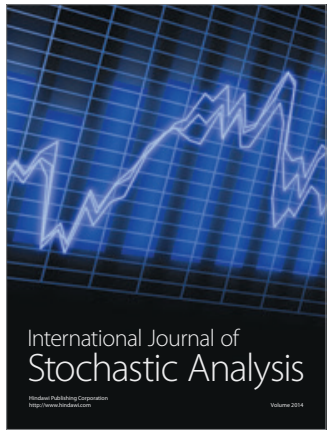

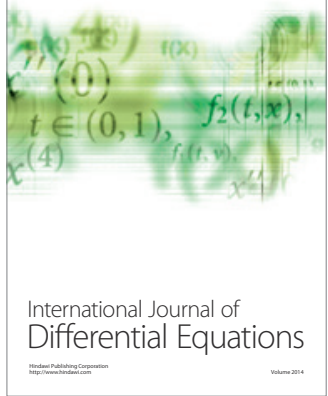
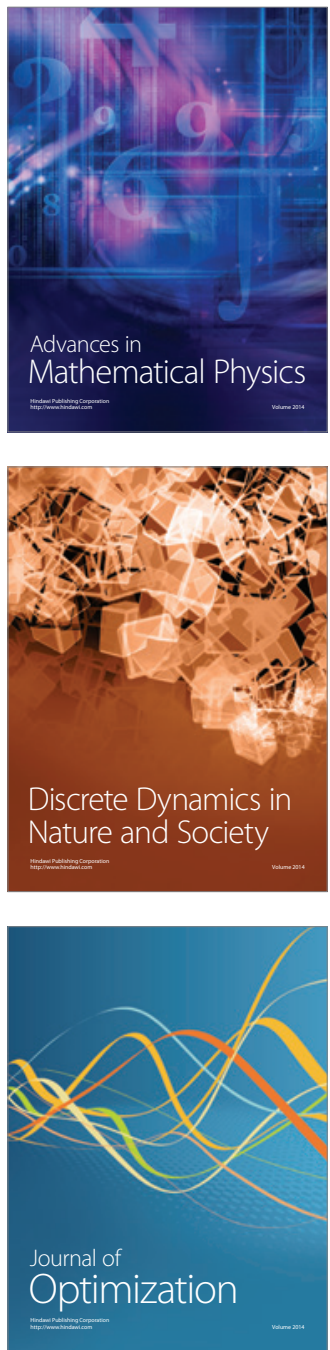